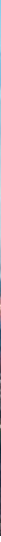

\title{
Fortbildungswoche München: Spürbare Fortschritte in der Dermatologie!
}

Ende Juli 2012 war es wieder soweit: die dermatologische Familie traf sich zur traditionellen Fortbildungswoche in München! Neue diagnostische und therapeutische Kenntnisse für die tägliche dermatologische Praxis wurden ausgetauscht. Ein Kompliment an die Veranstalter für das straffe und gleichzeitig anspruchsvolle Programm. Ja, sie hat sich weiterentwickelt - unsere FoBI! Neben den traditionellen Pharmaaustellern hat sich die kosmetikaassoziierte Industrie eingefunden. Überall spielt das Marketing die Hauptrolle! Beim Rundgang fielen einige neuere diagnostische Methoden auf, die Einzug in fortgeschrittene dermatologische Praxen halten: die konfokale Lasermikroskopie, die PC-gestützte Auflichtmikroskopie mit neuen Darstellungsmöglichkeiten suspekter Hautläsionen, die optische Kohäszenztomographie, um nur einige zu nennen. Frau Prof. Welzel aus Augsburg hat die Stärken und Schwächen der Geräte sehr gut herausgearbeitet.

Beim Thema Hauttumoren fallen die Fortschritte in der Diagnostik und Therapie maligner Geschwülste besonders ins Gewicht. Mit den neuen technischen Möglichkeiten sind die Fähigkeiten des Dermatologen gestiegen, dem Patienten durch eine noch qualifiziertere Diagnostik die Dignität eines Tumors prä Exzisionem zu belegen. Damit sind wir an der Schnittstelle zwischen Medizin und Ästhetik angekommen. Die Diagnostik gehört dabei zweifelsohne noch in die Heilkunde, die Entfernung einer gutartigen Geschwulst aus kosmetischer Indikation eher nicht. Je nach der weiteren Entwicklung des Gesundheitswesens unter der derzeitigen Sparwelle und Eurodiskussion wird sich im kommenden Jahr nach der Bundestagswahl entscheiden, ob das Institut eine Renaissance erlebt oder nicht. Man hört auch von Diskussionen Freiberufler generell der Gewerbesteuer zu unterziehen ...

Ein neuartiges und nicht wenig diskutiertes Instrument zur Besserung des Hautreliefs bei verschiedenen u.a. auch ästhetischen Indikationen ist der Derma-Roller ${ }^{\circledR}$. Bei der Demonstration auf der FoBi platzte der Hörsaal aus allen Nähten. Interessant war der Hinweis auf die Aufdeckung von Plagiaten mit ge-

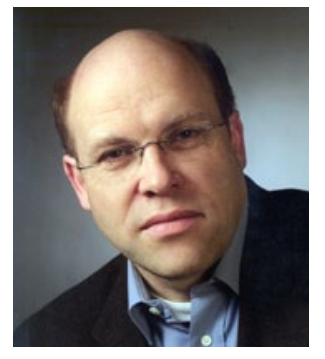

Dr. Matthias Herbst

fälschter CE-Kennzeichnung, die u.U. für eine problematische Haftungssituation für den Anwender sorgt.

Die kleinen Tipps und Neuheiten wurden am Schlusstag nochmals liebevoll vom Veranstalter Herrn Prof. Dr. Ruziska zusammengetragen. Unvergessen auch der Vortrag von Nana Schürer zur Kosmetik des Herrn, inklusive der bestellten Proteste der Männerseele durch Kollegen Zimmermann aus Heusenstamm!

Alles in allem ein sehr positives Fazit sowohl seitens der Besucher wie auch der Aussteller. Und der Veranstalter? Mehr als 4000 Besucher über alles gerechnet sprechen für sich.

Die klassische Dermatologie ist im Wandel. Man merkt es umgekehrt dürfen wir aber nicht vergessen, woher wir kommen. Die Basis unseres Tuns ist und bleibt die solide und seriöse Medizin.

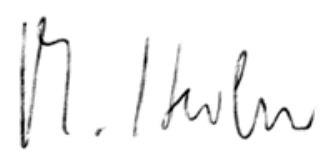

